\title{
Gobiernos panistas e inseguridad pública en Jalisco*
}

\author{
Alberto Arellano Ríos
}

El presente artículo hace un análisis sobre la inseguridad pública en Jalisco a partir de 1992. Se hace un recuento de las movilizaciones sociales suscitadas por el entorno de inseguridad en los gobiernos prístas y de cómo se dio la alternancia en Jalisco, para finalmente señalar que la inseguridad pública dejó de ser un factor de movilización para colocarse, al término del segundo gobierno panista, como un asunto de políticas públicas o, en el peor de los casos, de programas gubernamentales. La reflexión sobre las políticas de seguridad en la entidad concluye que éstas se diseñan y formulan sólo con la participación de dos actores: el gobierno estatal y los empresarios. De allí que sean limitadas y que respondan más a los intereses y posturas ideológicas de los segundos.

PALABRAS CLAVE: inseguridad pública, alternancia, empresarios, gobierno, PAN y políticas públicas

\section{Panista Administrations and Public Insecurity in Jalisco}

This paper analyses public insecurity in Jalisco since 1992. It chronicles the social demonstrations brought on by the public insecurity experienced during the Priísta administrations, and also the way in which electoral alternance was achieved in Jalisco. Finally it points out that public insecurity is no longer a factor of social protest and, during the final stages of the second Panista administration, it has become the object of public policies, or in the worst case scenario, of governmental programs. It concludes that only two types of participants are involved in the elaboration of Jalisco's security policies: the state's government and local businessmen, which is why such policies are limited in scope and tend to respond to the particular interests and ideological stances of the latter.

KEY WORDS: public insecurity, alternance, businessmen, government, PAN, public policies

AlberTo ARELlano Ríos: Universidad de Guadalajara, Guadalajara, Jalisco, México. betoarellano14@hotmail.com

*Agradezco las observaciones de los dictaminadores anónimos de Desacatos y de quien me auxilió en la corrección de estilo. 
$\mathrm{E}$ s curioso que en la década de 1990 en América Latina haya disminuido la violencia política y, en contraste, aumentado la violencia criminal y el temor a la delincuencia (Frühling, 2002). Una posible explicación puede ser que en la década de 1980 el fenómeno de la inseguridad pública no logró el reconocimiento como un problema grave que el Estado debía resolver, debido a que entre los diversos actores y sectores políticos, sociales y económicos se le dio prioridad al proceso de democratización. El objetivo de los actores políticos fue combatir y denunciar la violencia que el mismo Estado cometía y no la violencia social presente en la sociedad.

En este contexto en Jalisco, al igual que en América Latina, durante la década de 1990 los índices delictivos aumentaron considerablemente, pero en la entidad se dio una particularidad: el entorno de inseguridad se convirtió en un factor central para la movilización ciudadana, misma que incidió en el cambio político y permitió la alternancia. De esta forma, el fenómeno de la inseguridad pública fue un factor central que propició la llegada del Partido Acción Nacional (PAN) a importantes espacios del régimen locall.

Sin embargo, aunque el fenómeno de la inseguridad fue un factor que incidió en el cambio político en la primera mitad de la década de 1990, a principios del siglo XXI se vislumbró sólo como un fenómeno de políticas públicas. Así, en un primer momento fue un elemento que incentivó la alternancia política en Jalisco para pasar, en los últimos años, a ser sólo un asunto de acción gubernamental. En otras palabras, pese a que la inseguridad pública es un tema central en el núcleo duro de la agen-

\footnotetext{
${ }^{1}$ La noción de cambio político que aquí se utiliza es la que delinea Leonardo Morlino, 1995; por éste debemos entender el cambio de un régimen autoritario a uno más o menos democrático. Dicho cambio equivale a decir cambio del o en el sistema político y se da en todos o en algunos de sus componentes como son: la cultura, la estructura, los grupos, los leadership y las orientaciones políticas. El cambio político se puede comprender como un proceso político de larga duración y hoy, en el lenguaje de los estudios políticos, es conocido con el nombre de transición a la democracia. En su momento se creyó que la alternancia política en Jalisco profundizaría la democratización, pero nada más alejado de la realidad, pues después de dos gobiernos panistas el cambio ha sido parcial y en ocasiones hasta hubo retrocesos. El cambio político por lo tanto ha sido intermitente.
}

da de Jalisco, ya dejó de ser un factor de movilización en pos de la profundización del cambio democrático ${ }^{2}$.

Por ello, en las siguientes líneas se abordará la problemática que se presenta en torno a la seguridad pública en Jalisco desde la contraposición entre cómo su incumplimiento fue, en un primer momento, un elemento fundamental que propició el cambio político en Jalisco y cómo luego, en los gobiernos panistas, se circunscribió a ser sólo un asunto de políticas públicas. Dicho de otra forma, hay entonces en la primera mitad de la década de 1990 una relación empírica - mas no conceptual- entre inseguridad pública y cambio político en la entidad ${ }^{3}$.

Puesto que la inseguridad pública es una problemática compleja y a fin de mantener un control analítico, el eje articulador en la comprensión del fenómeno se establecerá a partir de dos actores centrales en el proceso de formulación de las políticas de seguridad: el gobierno del Estado y los empresarios. En esa relación serán descritos varios hechos coyunturales, los cuales conformarán un entorno de inseguridad pública y zozobra en la entidad. Éstos serán el contexto social del cambio político y de la alternancia. La secuencia lógica que tiene dicho contexto, vista a la distancia y a grandes rasgos, es la siguiente: primero, que el fenómeno de la inseguridad pública propició la movilización ciudadana en los gobiernos priístas; segundo, que tal movilización, junto con otros hechos sociales y políticos, devino en la alternancia en el gobierno de Jalisco; y tercero, en que un grupo de interés - los empresarios - fue - y es - el actor central en el diseño y formulación de las políticas de seguridad 4 .

En suma, el análisis considera la interacción de los

\footnotetext{
${ }^{2}$ Los otros temas del núcleo duro de la agenda de Jalisco al final del siglo XX y principios del XXI fueron el abastecimiento de agua a la zona metropolitana de Guadalajara, el desempleo y el transporte público. ${ }^{3}$ Diversos trabajos que han estudiado o evaluado el cambio político en Jalisco coinciden en señalar que la inseguridad pública fue un fenómeno, entre otros más, que propició la alternancia panista en Jalisco. Véanse, por ejemplo, los trabajos de Romero, 2001; Gómez López, 1997; y Alonso, 1995.

${ }^{4}$ El proceso de formulación de las políticas de seguridad debemos entenderlo como el cumplimiento de tareas críticas requeridas para resolver o atender el problema en cuestión. En él está inmersa la definición, precisión o delimitación del problema público por parte de los actores involucrados en el proceso de discusión y diseño de las políticas públicas.
} 


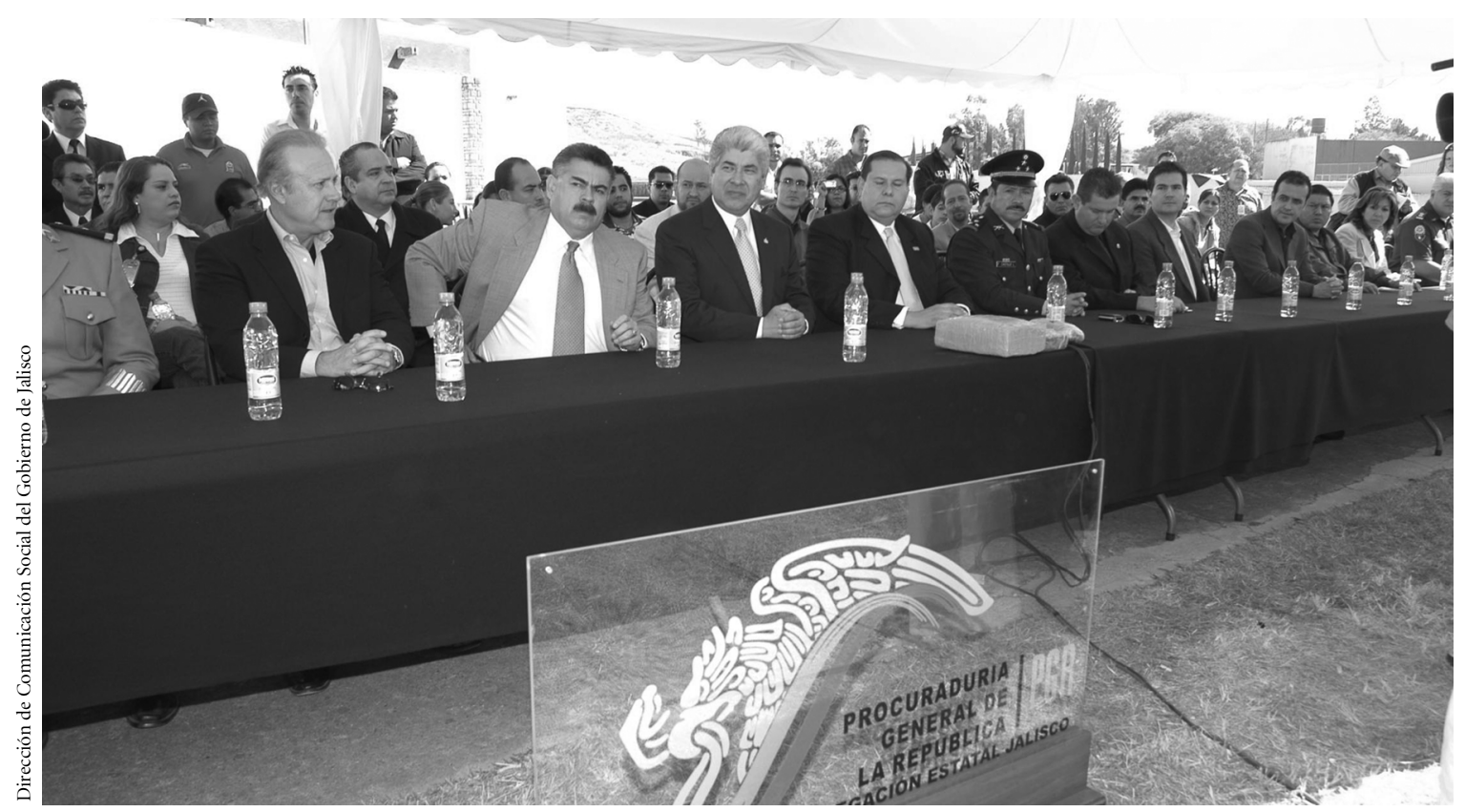

Reunión de trabajo y coordinación de seguridad jurídica entre la delegación de la PGR y los directores de Seguridad Pública de los ayuntamientos de la Zona Metropolitana de Guadalajara, encabezada por el gobernador del estado, Francisco Ramírez.

actores en el proceso de la formulación de esta política sectorial a cargo del Estado. En la discusión y formulación de las políticas públicas se puede comprender la forma en que interactúan los diversos actores en la arena política. Siendo más concreto, se observa cómo construyen y conciben un problema público, así como los alcances y limitaciones de solución y acción gubernamental. Por lo tanto, las agendas y la definición del problema son el elemento central en el análisis del fenómeno de la inseguridad pública. Sin embargo, al final éste será uno de los asuntos no resueltos por el gobierno de Jalisco, independientemente de su filiación partidista.

\section{DEFINIR LA INSEGURIDAD PÚBLICA EN MÉXICO}

La seguridad pública está vinculada a una idea primaria de orden público y es una función básica del Estado, misma que explica su propia génesis y existencia. Dicho en otros términos, no se entiende la intervención guberna- mental sin la garantía de la seguridad pública (la ley y el orden) en tanto razón de ser del Estado. Si la seguridad pública se correlaciona con la legislación penal que busca la convivencia social ordenada, segura, pacífica y equilibrada, la inseguridad pública se define en un sentido negativo. Así, ésta es la ausencia total o parcial de orden público, y en ella la convivencia social es desordenada, violenta e inestable.

Si bien en los últimos años del siglo XX se construyeron nuevos paradigmas de seguridad, en México persiste una idea clásica y decimonónica de la seguridad pública. Los paradigmas que se delinearon fueron cuatro: 1) el modelo integral de seguridad humana que impulsa la Organización de las Naciones Unidas (ONU); 2) el de seguridad regional o transnacional en bloques de integración económica; 3) el de seguridad ciudadana conformado en América del Sur en oposición al de seguridad nacional, cuyo ente de defensa de seguridad y defensa era el Estado y no el individuo, y 4) el modelo local que respondió a la relación global-local y que entraría dentro de los modelos policiales comunitarios (Moloeznik, 2002). 
Así, se ha tratado de superar - pero no abandonarla idea de orden público. Con base en esta idea, la delincuencia y la inseguridad pública han sido estudiadas desde siempre por la criminología, el derecho penal o desde los enfoques policiacos - disciplinas con amplia tradición-, pero en la vertiente del crimen y el castigo. Sin embargo, en América Latina, desde la década de 1980 varios investigadores y estudiosos del fenómeno de la inseguridad han intentado explicarlo y comprenderlo a partir de dos grandes enfoques: a) desde una perspectiva institucional, y b) desde un disfuncionalismo social estructural.

El primer enfoque indaga sobre las fugas que el sistema de seguridad pública tiene en su interior. Intenta descubrir cuáles son los incentivos formales e informales que hay en las organizaciones e instituciones públicas que provocan la ineficacia institucional del Estado en su lucha contra la delincuencia. La "jurimetría”, el neo-institucionalismo, la sociología de las organizaciones, el análisis de políticas públicas y la gerencia pública en el estudio de la inseguridad pública son las propuestas concretas que se pueden enmarcar en este enfoque.

122 El segundo ve a la inseguridad pública como un problema estructural en la sociedad. Intenta descubrir las fallas concretas de desigualdad estructural que provocan este fenómeno. Entiende que hay causas internas que producen procesos de desintegración y desarticulación social que establecen relaciones imperfectas, de ahí que los procesos de socialización sean esenciales para comprender la inseguridad. La falta de seguridad y la violencia social que aumentó con los desmantelamientos de los Estados latinoamericanos son, en buena medida, el factor central de explicación. Así, el adelgazamiento del Estado no trajo consigo la funcionalidad institucional y organizativa que la ideología neoliberal presuponía, sino que contribuyó aún más al estallido de fenómenos sociales como la pobreza y la violencia en América Latina (Arraigada y Godoy, 1999; y Buvinic et al., 1999).

Sin embargo, en México, entre los actores políticos, sociales y económicos priva una idea decimonónica sobre la seguridad. La explicación está en la tradición jurídica positiva mexicana que reduce el orden público al cumplimiento de la ley. Dicha tradición parte de suponer que, por el carácter coercitivo de ésta y a través del uso de la fuerza, es posible mantener el orden y disipar las conductas antisociales y criminales. Consecuentemente, el acto policiaco se identifica entonces con el poder político y es la forma en que penetra en la sociedad civil para servir al interés de una idea de justicia y de orden público (Cohen y Arato, 2000: 133). De esta manera, en el Estado mexicano está más presente el elemento represivo contra las manifestaciones que puedan trastornar las relaciones político-económicas que la idea de concebir a la seguridad pública como un entorno o escudo para el desarrollo integral del individuo y la sociedad. El orden público se limita, por lo tanto, al uso de la fuerza en sus modalidades de disuasión y castigo.

Esto explica que el elemento preventivo y participativo de la ciudadanía en materia de seguridad pública sólo se encuentre en el nivel discursivo de las políticas y los programas gubernamentales. En consecuencia, existe un desfase entre las exigencias sociales resultantes por la inseguridad pública y los instrumentos institucionales e ideológicos por medio de los cuales se guía el Estado mexicano. Aún más, en los años recientes incluso el discurso de una seguridad ciudadana se está haciendo a un lado de forma burda. En los hechos, el Estado mexicano, al verse rebasado por el narcotráfico, está reforzando prácticas represivas. El uso del Ejército en labores de seguridad pública en el gobierno del presidente Felipe Calderón corrobora lo anterior y es la muestra más evidente del fracaso de las políticas de seguridad pública de, por lo menos, los tres últimos gobiernos federales.

No obstante, en el campo académico mexicano se han estado haciendo trabajos que no se basan en el enfoque clásico del derecho y la victimología y, por lo tanto, que rebasan la "visión judicialista de la inseguridad pública". Pese a esta emergencia, aún no existe una cartografía analítica de los mismos ${ }^{5} \mathrm{y}$, ni mucho menos, los tomadores

\footnotetext{
${ }^{5}$ Distintos investigadores han retomado en algún momento las inquietudes hasta ahora planteadas. A partir de distintas disciplinas académicas y en varios artículos, ensayos y trabajos de investigación se observa que el complejo fenómeno de la inseguridad pública adquiere mayor comprensibilidad. Por ejemplo, entre los exponentes de estas nuevas líneas de investigación están Marcos Pablo Moloeznik, Luis Astorga, Guillermo Zepeda Lecuona, Jorge Regalado, Juan Manuel Ramírez Sáiz, Alberto Chávez Sevilla y María Eugenia Suárez de Garay; o ins-
} 
de decisiones se han apropiado o interpretado los resultados a los que han llegado. Construido este piso conceptual, el artículo retoma la idea de analizar las políticas de seguridad pública en los gobiernos panistas de Jalisco. La intención es que algunas ideas o enfoques teóricometodológicos puedan ser retomados para otras experiencias en México o en América Latina.

\section{LA INSEGURIDAD PÚBLICAY EL CAMBIO POLÍTICO}

El entorno de inseguridad pública que hubo en Jalisco durante los años previos a la alternancia de 1995 se manifestó en los siguientes hechos: a) el aumento generalizado de los índices delictivos; b) la apropiación de la capital del estado por el narcotráfico; c) el asesinato de Ivette Roux, que desencadenó el movimiento colectivo de Madres Unidas Contra la Violencia o Mujeres de Negro; d) las explosiones del 22 de abril de 1992; e) el asesinato del cardenal Juan Jesús Posadas Ocampo, el 24 de mayo de 1994, y f) la explosión de un coche-bomba a las afueras del hotel Camino Real, por mencionar sólo los más importantes.

La situación social que se suscitó a partir de los sucesos anteriores conformó un entorno de irritación ciudadana en Jalisco que propició la alternancia. Tal contexto político y social en lo particular incentivó una de las acciones colectivas más significativas. Diversas organizaciones civiles y sociales de las más distintas posiciones políticas e ideológicas convocaron a una marcha el domingo 6 de junio de 1993, bajo el lema de "Una sola voz” (Regalado, 2001). Esta acción colectiva marcó el clímax de la insatisfacción ciudadana por la inseguridad pública que en la entidad se vivía. Los manifestantes y las diversas organizaciones que se unieron en ella se pronunciaron en los siguientes términos:

1. Manifestamos nuestro profundo pesar por el asesinato del señor cardenal Juan Jesús Posadas Ocampo y de seis personas.

tituciones y organismos como el Centro de Investigación y Docencia Económicas (CIDE), el Centro de Investigación para el Desarrollo, A.C. (CIDAC), el Instituto Ciudadano para el Estudio de la Seguridad, por mencionar algunos.
2. Repudiamos la situación de violencia e inseguridad que prevalece en el estado de Jalisco.

3. Denunciamos la incapacidad y corrupción de algunas autoridades, tanto para acabar con la delincuencia como para controlar a sus propias corporaciones policiacas.

4. Exigimos a las autoridades que se nos diga la verdad acerca de todos los asuntos que, como el pasado día 24, son de interés de la población.

5. Exhortamos a la ciudadanía a que pase del dolor a la acción, de la queja a la participación activa, de la denuncia a la proposición de alternativas. Hagamos valer así nuestros derechos, rescatemos nuestra dignidad 6 .

La situación de irritación ciudadana provocada por los hechos anteriores, conjuntamente con el error de diciembre que provocó la devaluación del peso y una fuerte crisis económica, así como la actitud del presidente Zedillo para no permitir el fraude, facilitó que el 12 de febrero de 1995, Alberto Cárdenas Jiménez ganara la gubernatura de Jalisco. Cárdenas Jiménez no llegó sólo al poder sino que su partido, el PAN, ganó las cuatro presidencias municipales de la Zona Metropolitana de Guadalajara (ZMG), otros cincuenta ayuntamientos de Jalisco y la mayoría parlamentaria en el Congreso local. Con el triunfo de los panistas, la ciudadanía manifestó su inconformidad con los ya desgastados mecanismos de interacción del viejo régimen local del Partido Revolucionario Institucional (PRI). En este escenario y por el malestar ciudadano, vía el voto de castigo, fue posible una alternancia pragmática y no programática (Alonso, 1995: 206-208).

Contrario a lo que se pudiera pensar, la inseguridad pública en el gobierno de Alberto Cárdenas no disminuyó, sino que los índices delictivos tuvieron un crecimiento generalizado que por momentos pareció alarmante. Las mismas cifras oficiales corroboraron esta tendencia y fortalecieron la percepción social de que el gobierno de Jalisco estaba siendo rebasado por la delincuencia (véase cuadro 1, p. 124).

Así, el "trastocamiento" del régimen que trajo consigo la alternancia panista en Jalisco fue aprovechado por la delincuencia. Y la inseguridad se manifestó primariamente

\footnotetext{
6 "Por la vida, por la verdad..., ¡Una sola voz! Marcha familiar silenciosa", Siglo 21, Guadalajara, 6 de junio de 1993, p. 12.
} 
Cuadro 1. Delitos denunciados en Jalisco (1995-2005)

\begin{tabular}{l|r|r|r|r|r|r|r|r|r|r|r}
\hline & 1995 & 1996 & 1997 & 1998 & 1999 & 2000 & 2001 & 2002 & 2003 & 2004 & 2005 \\
\hline Robo de vehículos & 15876 & 18661 & 19904 & 16264 & 14705 & 14035 & 13474 & 10779 & 8760 & 6570 & 5840 \\
\hline Robos a casa habitación & 5358 & 6518 & 6120 & 5223 & 5400 & 5133 & 6345 & 6694 & 6427 & 5032 & 5773 \\
\hline Robo a negocios & 6649 & 7748 & 6585 & 5405 & 5637 & 5276 & 6931 & 6557 & 6071 & 4753 & 5693 \\
\hline Robo a autotransportes & 224 & 317 & 747 & 860 & ND & ND & 893 & 719 & 555 & 492 & ND \\
\hline Asaltos bancarios & 69 & 81 & 55 & 39 & 50 & 55 & 18 & 6 & 11 & 11 & 2 \\
\hline Abigeato & 290 & 319 & 550 & 481 & 448 & 366 & ND & ND & ND & ND & ND \\
\hline Secuestros & 55 & 45 & 112 & 49 & 32 & 26 & 14 & 16 & 13 & 13 & 5 \\
\hline Homicidios dolosos & 678 & 670 & 801 & 612 & 518 & 442 & 488 & 431 & 425 & 370 & 375 \\
\hline
\end{tabular}

Fuente: Informes de Gobierno de Alberto Cárdenas Jiménez y Francisco Ramírez Acuña. ND: No hay dato.

en el aumento de los delitos denunciados que recibió el gobierno del estado por medio de la Procuraduría de Justicia. En ellos fue notorio que en los tres primeros años del primer gobierno panista, los delitos con mayor número de denuncias correspondieron al robo de vehículos, seguidos por los asaltos bancarios 7 . Pero además, la cifra de secuestros creció de manera alarmante. Estos últimos delitos fueron el dolor de cabeza y causa de la presión política más fuerte en la gestión de Alberto Cárdenas.

Los asaltos bancarios alcanzaron su nivel máximo en 1996 con 81 denuncias; el robo de vehículos en 1997, con 19904 casos reportados; el mayor número de robos a autotransportes se dio en 1998, con 860 incidencias. Pero sobre todo, el secuestro alcanzó su nivel máximo y más escandaloso en 1997, con 112 casos. Entre las personas secuestradas hubo connotados empresarios locales (más adelante señalaremos quiénes fueron). La alarma social por esta ola de secuestros será una de las causas que provocó el posicionamiento público de los organismos empresariales en torno a las políticas de seguridad llevadas a cabo hasta ese momento.

Además del aumento de los índices delictivos, en el juego sociopolítico el entorno de inseguridad pública le

\footnotetext{
${ }^{7}$ Toda medición de un fenómeno social causa un debate sobre su precisión. Los especialistas hacen énfasis y tratan de medir la "cifra negra" de los delitos, es decir, aquellos que no son denunciados. No obstante, la denuncia es una expresión que indica un contexto social de inseguridad concreto.
}

significó al gobierno de Alberto Cárdenas que el fenómeno ya no fuera sólo un problema social que el Estado debía controlar, sino que se convirtiera en un problema en sí político. Es decir, el fenómeno se trastocó en un elemento instrumental de organización, movilización, protesta y posicionamiento público de varios sectores de la sociedad en torno a las políticas públicas. Principalmente por parte de un empresariado, respecto al cual y de acuerdo con su visión, se formularon y diseñaron las políticas en la materia.

De esta forma, los delitos que preocuparon a los grupos y actores empresariales originaron una tendencia muy marcada hacia la privatización de la seguridad pública. El secuestro tradicional ${ }^{8}$, el robo de vehículos y los asaltos bancarios desencadenaron la proliferación de empresas privadas que ofrecieron servicios de seguridad. Esta manifestación del mercado se conoce desde entonces como la industria del miedo (Regalado y Moloeznik, 1998).

En contraste con el gobierno de Alberto Cárdenas, la administración de Francisco Ramírez Acuña obtuvo mejores resultados, según las cifras oficiales (véase cuadro 1). Los delitos que anteriormente constituyeron el "dolor de cabeza” de la gestión gubernamental, como el secuestro

\footnotetext{
${ }^{8} \mathrm{La}$ aparición del secuestro express evidencia la capacidad con la que la delincuencia se transforma, readecua y responde a las medidas que el Estado implementa; esta situación obliga siempre a las autoridades a formular nuevas estrategias pero cada vez en función de lo que el crimen marca como pauta.
} 
Cuadro 2. El secuestro en México y Jalisco

\begin{tabular}{l|c|c|c|c|c|c|c|c|c|c|c}
\hline & 1995 & 1996 & 1997 & 1998 & 1999 & 2000 & 2001 & 2002 & 2003 & 2004 & 2005 \\
\hline México* $^{*}$ & 547 & 569 & 1047 & 734 & 590 & 548 & 505 & 433 & 422 & ND & ND \\
\hline Jalisco $^{* *}$ & 55 & 45 & 112 & 49 & 32 & 26 & 14 & 16 & 13 & 13 & 5 \\
\hline
\end{tabular}

Fuente: ${ }^{\star}$ Ocho Columnas, Guadalajara, 18 de febrero de 2004, p. 1B. ${ }^{* \star}$ Informes de Gobierno.

ND: No hay dato.

y los asaltos bancarios, disminuyeron drásticamente. El número de delitos reportados fue el más bajo desde 1995, salvo el de robo a casas habitación, robo a negocios y robo de mercancías a autotransportes.

Así, las incidencias delictivas más altas en materia de secuestro, durante la gestión de Ramírez Acuña, se dieron en el año 2001 con 16 casos; y descendieron a cinco en 2005, la cifra más baja hasta ese momento. Los asaltos bancarios, por otro lado, también tuvieron un comportamiento sensible a la baja. De once asaltos en el año 2003 se llegó a la cifra de cinco en 2005. Pero además, el segundo gobierno del PAN en Jalisco presentó los mejores resultados en el combate al secuestro en comparación con el ámbito nacional (véase cuadro 2). Esto según las cifras proporcionadas por las dependencias de seguridad y de procuración de justicia.

Aparentemente, Ramírez Acuña entregó buenas cuentas en el rubro de seguridad pública. No obstante, los problemas en su gobierno derivados del entorno de inseguridad pública se dieron más en la violación de los derechos humanos como producto de su "estilo personal de gobernar"9.

\footnotetext{
${ }^{9}$ Hasta el 31 de agosto de 2006 el gobierno de Francisco Ramírez contaba con 753 quejas presentadas ante la Comisión Estatal de Derechos Humanos, principalmente por tortura. En contraste, el gobierno de Alberto Cárdenas sólo fue denunciado en 57 ocasiones. Véase "Llueven críticas a la postura oficial sobre quejas de tortura", Público, Guadalajara, 19 de septiembre de 2006, pp. 6-7. Amante del orden, a Francisco Ramírez se le reconoció "su oficio político". A diferencia de su antecesor, él fue "el eje en torno al cual se reúnen las fuerzas vivas de los distintos partidos políticos en el estado". No le molestó relacionarse con gente del PRI; su pragmatismo político fue inversamente proporcional a la postura ideológica de Alberto Cárdenas Jiménez, que parecía odiar todo lo priísta. El objetivo de Francisco Ramírez era simple: mantener la paz y la estabilidad. Mantuvo relaciones aun con opositores políticos e ideológicos, que en algunos casos fueron de amistad. La mayoría de las relaciones con priístas fueron cultivadas a partir de su
}

Recapitulando, aunque el gobierno de Ramírez Acuña presentó mejores resultados en seguridad pública, según las cifras oficiales, la percepción ciudadana objetó dichos logros. Pese a sus resultados, la discusión se dio en otros rubros: en el plano de la percepción. Se concluyó entonces que la inseguridad pública, la percibida por la ciudadanía y la real, aumentó en los gobiernos del PAN (más adelante será retomada esta discusión).

$\mathrm{Al}$ respecto, se llegó a tolerar a los dos gobiernos panistas, o más bien se conformó un factor de explicación de tal situación: éste señala que la situación de inseguridad pública se debió a un proceso de reajuste entre un viejo y nuevo orden. De esta forma, y según esta explicación, el aumento de dichos índices fue más un producto del reacomodo entre los grupos criminales, las fuerzas políticas y los cuerpos policiacos y militares que estaban coludidos con la delincuencia organizada ${ }^{10}$. La percepción de que el PAN no fue el responsable directo del desorden y del aumento de la inseguridad pública le conservó en ese momento el apoyo ciudadano. Es así como la inseguridad dejó entonces de incentivar el cambio político y el fenómeno se situó como un asunto de agenda y acción gubernamental: esto es, de políticas públicas ${ }^{11}$.

participación en la XLIX Legislatura estatal (1980-1983). Véase "Los amigos prístas del gobernador", Público, Guadalajara, 14 de mayo de 2002, en: <www.milenio.com>.

${ }^{10}$ Destacan en este punto el asunto del general Jesús Gutiérrez Rebollo y Horacio Montenegro. Véase Preston y Dillon, 2004: 207-228.

${ }^{11} \mathrm{El} \mathrm{ABC}$ de las políticas públicas se puede comprender en tres principios básicos: a) las políticas públicas se explican por su intención de resolver un problema o problemas catalogados como públicos que merecen la intervención gubernamental; b) las políticas públicas son decisiones gubernamentales que incorporan la mayor participación ciudadana posible, y c) en el proceso de formulación y ejecución de las políticas públicas hay ganadores y perdedores. 


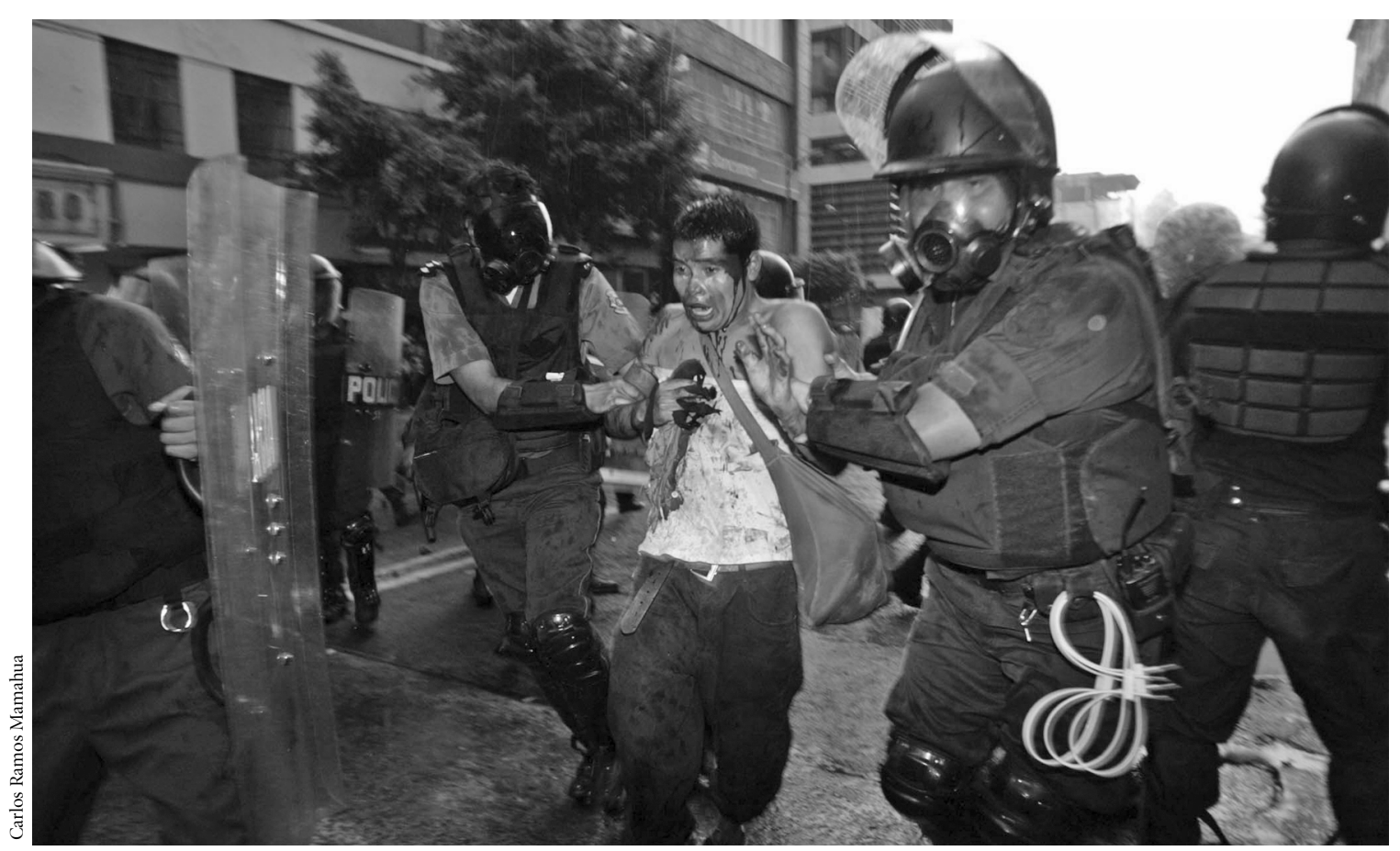

Golpes contra manifestantes del 28 de mayo de 2004, Guadalajara.

De ser cierta esta hipótesis, el fenómeno de la inseguridad pasa a situarse más como un tema en el que la discusión no gira en torno al cambio o la transformación del orden social y político sino en dar respuesta a una demanda concreta. Esta idea se fortalece cuando amplios actores y sectores sociales que demandaron mayor seguridad y criticaron, al mismo tiempo, los ineficientes resultados de los gobiernos panistas, toleraron sus acciones $y$ proceder. Una actitud muy distinta a la mostrada ante los gobiernos priístas.

La alternancia panista que se dio en 1995, si bien se percibió como el inicio de un cambio político profundo, tras dos gobiernos de este partido ha sido vista más como un fenómeno que instaura, reproduce o hace circular gobiernos predemocráticos o inciviles. Así, la alternancia puso sobre la mesa de reflexión el hecho de que los gobiernos del PAN no han logrado transformar ni dejar por completo los antiguos mecanismos de negociación y legitimación del poder político (Regalado, 2005).

En suma, la alternancia en Jalisco no se tradujo en un cambio en las hechuras de las políticas públicas, pues más bien se suscitó un cambio político sin un cambio en la forma de formular las políticas públicas (Acosta Silva, 2004: 54-55). Por lo tanto, la formulación de las políticas públicas en materia de seguridad será entonces el siguiente punto a abordar.

\section{POLÍTICAS PÚBLICASY ACCIÓN GUBERNAMENTAL}

El fenómeno de la inseguridad pública como asunto de políticas públicas se sitúa en tres espacios. El primero es aquel en el que debió ser discutido y definido como un problema público. En esa discusión es en la que se va delineando la forma cómo la acción gubernamental resuelve o da respuesta a cierta demanda social. El segundo espacio es el de la formulación de las políticas de seguridad, en el que se recrea un proceso de lucha y negociación entre actores, no estrictamente por el poder sino por la co- 
locación de intereses, valores y sentimientos en la acción gubernamental. El tercer y último espacio es el de la concretización de las políticas de seguridad pública en programas y recursos públicos. En este espacio es importante tener en cuenta el marco normativo, la forma en que se distribuyen y utilizan los recursos financieros, así como el desenvolvimiento de las burocracias.

El eje articulador del análisis en el proceso de formulación de las políticas de seguridad pública en Jalisco señala que un solo actor fue el que más peso tuvo: los empresarios. Otros actores e intereses importaron en la medida en que respondieran al perfil ideológico del empresariado. Así, el monopolio discursivo y político que los organismos de la iniciativa privada tuvieron sobre el fenómeno de la inseguridad estimuló un diseño de las políticas de acuerdo con las visiones que ésta tenía de él.

De esta forma, se puede sostener como idea central que la legitimidad de las políticas de seguridad en Jalisco no estuvo en sus resultados sino en el proceso instrumental de su formulación. Esto es: que la legitimidad de las politicas de seguridad pública está garantizada si en el proceso de formulación y diseño sólo se incluye al gobierno estatal y a un grupo específico relevante: los empresarios, agrupados básicamente en tres organismos: la Confederación Obrero Patronal Mexicana (Coparmex), la Cámara Nacional de Comercio de Guadalajara (Canaco) y el Centro Empresarial de Jalisco (CEJ). Así, independientemente de si resuelven o no el problema de la inseguridad, la respuesta y la acción gubernamental sólo han sido encaminadas a atender a un grupo social.

\section{El empresariado como un actor clave}

Los grupos empresariales, en tanto que organizaciones corporativas, siempre hicieron suya la representación de la sociedad y en su nombre, a lo largo de la historia de Jalisco, suscribieron acuerdos, programas, pactos y planes con el poder político. Por lo tanto, la alternancia panista de 1995 no trastocó los antiguos mecanismos que el empresariado tapatío mantuvo con los gobiernos del estado. Al contrario, los fortaleció y los hizo públicos. Aun cuando hay una explicación histórica y cultural de dicha cercanía, ésta se construyó básicamente por la intermediación de la jerarquía católica. De esta manera se conformó un sistema de relaciones políticas y sociales de tipo endogámico que articularon más a una élite en Jalisco que a un conglomerado de clases o grupos sociales ${ }^{12}$.

Éste fue entonces el contexto sociohistórico que envolvió al subsistema de las políticas de seguridad pública en Jalisco $^{13}$. Desde las posiciones construidas a lo largo de la historia en la entidad es que el empresariado se ha presentado ante los gobiernos panistas como el principal actor articulador de la presión por el entorno de inseguridad. Los empresarios que en el régimen prísta habían sido excluidos de la participación política formal, ahora ya son parte importante en la toma de decisiones.

Desde la década de 1980 los empresarios jaliscienses se planteaban su participación formal en la política, pero no lo hicieron sino hasta la década de 1990, debido a que el escenario de apertura democrática les otorgó un nuevo papel en la arena pública. En él, los empresarios buscaron influir abierta y públicamente en las decisiones y las políticas gubernamentales.

A partir de las nuevas relaciones concretas que se dieron por la pluralización política, los empresarios de Jalisco han tratado de presentarse como un sector con la capacidad persuasiva y negociadora propia de un grupo de interés. Esto explica mejor el papel que han tenido en el proceso de formulación, diseño e implementación de las políticas de seguridad en Jalisco ${ }^{14}$. Así, el empresaria-

\footnotetext{
12 El empresariado tapatío ha sido estudiado por diversos investigadores, entre ellos: Olveda, 1991; Alba y González, 1989; Alba y Kruijt, 1988; y Hernández, 2002; por mencionar algunos.

${ }^{13}$ El subsistema de políticas es el "espacio político" de interacción entre el gobierno y los diversos grupos de interés. En este espacio se elabora la agenda gubernamental, se formulan las opciones de políticas y se discute el cómo. En él se dan las relaciones de poder entre los actores involucrados y también se discuten los asuntos "técnicos" de las políticas. Véase Aguilar Villanueva, 1996: 49.

${ }^{14}$ El empresariado en este nuevo escenario es más un grupo de presión porque moviliza, protesta o posiciona sistemáticamente sus demandas frente a la acción gubernamental. Los grupos de presión son parte significativa del proceso político en los sistemas democráticos, aunque no se les acepte plenamente. Pero cuando los grupos de presión son el factor determinante y explicativo del sistema político lo que se evidencia es una grave crisis del sistema de la administración pública y de los organismos representativos de la democracia. Véase Pasquino et al., 1986: 179-215.
} 
Cuadro 3. Empresarios en el gobierno de Jalisco

\begin{tabular}{|c|c|c|c|}
\hline Gobernador & Empresario & Procedencia & Cargo \\
\hline \multirow{2}{*}{$\begin{array}{l}\text { Enrique Álvarez } \\
(1983-1989)\end{array}$} & Gabriel Covarrubias Ibarra & Inmobiliarias & Tesorero general \\
\hline & Pedro Jiménez González & Restaurantero & Jefe de Planeación \\
\hline \multirow{2}{*}{$\begin{array}{l}\text { Guillermo Cosío } \\
(1989-1992)\end{array}$} & Ignacio Montoya González & Industria textil & Secretario de Finanzas \\
\hline & Enrique Dau Flores & Industria de la construcción & Secretario de Desarrollo Urbano \\
\hline \multirow{3}{*}{$\begin{array}{l}\text { Carlos Rivera } \\
(1992-1995)\end{array}$} & Gustavo Martínez Guitrón & Grupo Sidek & Secretario de Promoción Económica \\
\hline & Carlos González Lozano & Industria zapatera & Diputado y secretario de Turismo \\
\hline & Eugenio Pelayo López & Industria hulera & Secretario de Vialidad \\
\hline \multirow{6}{*}{$\begin{array}{l}\text { Alberto Cárdenas } \\
(1995-2001)\end{array}$} & José Levy García & Coparmex & Secretario de Finanzas \\
\hline & Sergio García de Alba & Canacintra & Secretario de Promoción Económica \\
\hline & Francisco Mayorga Castañeda & $\mathrm{CAJ}$ & Secretario de Desarrollo Rural \\
\hline & Pablo Gerbert Stump & Canaco & Secretario de Turismo \\
\hline & Carlos Petersen Biester & Industria de la construcción & Secretario de Desarrollo Urbano \\
\hline & Leopoldo Montelongo Jiménez & $\mathrm{CICJ}$ & Secretario de Vialidad \\
\hline \multirow{5}{*}{$\begin{array}{l}\text { Francisco Ramírez } \\
(2001-2007)\end{array}$} & Guillermo Martínez Mora & Coparmex & Secretario de Administración y secretario de Educación \\
\hline & José Luis Macías Romano & $\mathrm{CICJ}$ & Secretario de Desarrollo Urbano \\
\hline & Abraham González Uyeda & Sello Rojo & $\begin{array}{l}\text { Secretario de Promoción Económica y secretario de } \\
\text { Administración }\end{array}$ \\
\hline & Horacio González Pardo & Canaco & Secretario de Turismo \\
\hline & Enrique Dau Flores & Industria de la construcción & Comisión Estatal del Agua y Saneamiento \\
\hline
\end{tabular}

FUENTE: Elaboración propia con base en "El gobierno estatal con sello de sociedad anónima", Público, Guadalajara, 17 de julio de 2001, en <www.publico.com.mx>. CICJ: Cámara de la Industria de la Construcción de Jalisco.

do ha tenido un estilo discursivo y una acción muy particular que ha moldeado a su modo las políticas de seguridad. Con esta actitud alteraron las estructuras políticas y asumieron el papel de actor denunciante que exigía resultados por el entorno de inseguridad.

El gobierno de Guillermo Cosío (1989-1992) abrió espacios a los empresarios en puestos de primer nivel de la administración estatal. Éstos convergieron con otros espacios ganados en las administraciones municipales, el Congreso de Jalisco y los partidos políticos. Ya anteriormente lo había hecho el ex gobernador Enrique Álvarez del Castillo (1983-1989), pero no en puestos de secretarios de Estado. La misma línea siguió Alberto Cárdenas cuando designó a seis empresarios en importantes puestos de su gabinete. Finalmente, Francisco Ramírez concedió cuatro secretarías a los empresarios (véase cuadro 3).

El empresariado, fiel al objetivo de lograr una mayor y activa participación en el sistema político local, mostró su preponderancia sobre otros actores y sectores sociales. El peso socioeconómico y el reconocimiento institucional por parte del Estado hicieron que sus pronunciamientos públicos, demandas y propuestas tuvieran "mayor peso" que las de otros actores. Las autoridades valoraron que sus demandas y exigencias debían ser atendidas y resueltas inmediatamente. Los preferidos del gobierno para participar en actos públicos y dialogar en privado fueron los empresarios.

Un dato que ilustra lo anterior es el hecho de que los gobernadores Alberto Cárdenas y Francisco Ramírez casi no recibieron a puerta cerrada a las distintas agrupaciones campesinas, populares, sindicales, ciudadanas o civiles y dedicaron poco tiempo, o el mínimo requerido, a los representantes de los otros poderes (legislativo, judicial, gobierno federal y ayuntamientos, sin importar el origen partidario). Por ello no es casualidad que el sector empresarial fuera, y siga siendo, el grupo social que 
Cuadro 4. Gasto público en seguridad pública durante los años de la reingeniería institucional*

\begin{tabular}{l|c|c|c|c}
\hline & 1998 & 1999 & 2000 & 2001 \\
\hline Presupuesto total ejercido & 14548.92 & 18850.63 & 24999.95 & 27971.89 \\
\hline A seguridad & 797.28 & 1299.14 & 1530.80 & 1012.65 \\
\hline Porcentaje & $5.48 \%$ & $6.89 \%$ & $6.22 \%$ & 1801.36 \\
\hline
\end{tabular}

FUENTE: Informes de Gobierno. Cantidades en millones de pesos.

* Sólo se considera el rubro expresamente denominado Seguridad Pública, en él no se toma en cuenta las asignaciones presupuestales al Poder Judicial en materia penal y a la Procuraduría de Justicia del Estado, que forman parte del sistema de seguridad pública.

más influyó y participó en las decisiones de los gobiernos panistas. Al espacio que tenía ganado en el Consejo del Comité de Adquisiciones de la Secretaría de Administración (órgano encargado de vigilar las compras del gobierno del Estado ${ }^{15}$ ), se sumó el hecho de que los empresarios estaban representados en 13 de los 17 consejos consultivos que las dependencias públicas implementaron. La consigna de "atender y promover la participación” era comprendida únicamente como la participación activa del empresariado ${ }^{16}$.

En el caso particular de la participación ciudadana en materia de seguridad pública, todo indica que aun cuando los gobiernos aquí tratados de Acción Nacional se interesaban en ella, al menos discursivamente, no pudieron consolidarla por las vías institucionales. Por ejemplo, en el Consejo Ciudadano de Seguridad Pública del Estado hubo una sobre-representación de una perspectiva

${ }^{15}$ El Comité de Adquisiciones se concibió como un organismo integrado por representantes del sector privado y público para vigilar y determinar las compras de bienes y servicios.

${ }^{16}$ Entre los consejos en los que estuvieron representados los empresarios se encontraron: el Instituto Jalisciense de Asistencia Social; el Colegio de Estudios Científicos y Tecnológicos; el Consejo Estatal para el Fomento Deportivo; el Instituto de Fomento al Comercio Exterior, que incluye también universidades públicas y privadas; el Consejo Estatal de la Secretaría de Promoción Económica, que incluye también a líderes sindicales; el Consejo Estatal de Transplantes de Órganos y Tejidos; el Consejo Estatal de la Secretaría de Desarrollo Urbano; el Consejo Estatal para la Prevención de Accidentes; el Consejo Ciudadano de la Comisión Estatal de Derechos Humanos; el Consejo de Seguridad Pública, Prevención y Readaptación Social; el Consejo Estatal de la Familia. Hay otras instituciones en cuya estructura el gobierno considera la participación ciudadana, pero en realidad sólo participan funcionarios públicos: el Instituto Jalisciense de Antropología e Historia, el Consejo Estatal de Población y el Consejo Estatal Editorial. Véase "Privilegian a empresarios en consejos ciudadanos", Mural, Guadalajara, 6 de agosto de 1999, en <www.mural.com>. y un enfoque únicos en materia de seguridad. De los 24 integrantes que componen dicho consejo, 19 compartían en los hechos una misma visión del fenómeno ${ }^{17}$. De esta manera se conformó un consejo que no reflejaba la pluralidad social y política de la entidad y que vio la problemática de la inseguridad pública desde la perspectiva del orden público clásico: judicialista y criminológico. Por consiguiente, el asunto de los derechos humanos no fue tomado en cuenta como la base fundamental en la formulación de las políticas de seguridad pública.

De lo anterior se deriva la conclusión de que en Jalisco hasta 2006 los gobiernos del PAN apelaban a la participación ciudadana, pero en los hechos se observó que ésta fue parcial e impulsada en sectores afines y cómodos. Las formas principales de participación que los gobiernos panistas esperaron de la población fueron básicamente de colaboración y relativas a la proporción de información sobre delincuentes, sospechosos o posibles infractores, pero no de rendición de cuentas y/o coparticipación.

Así, el proceso de liberalización —que devino en democratización desde la mitad de la década de 1980puso poco a poco en la mesa de discusión en la década siguiente el tema de la inseguridad desde dos perspectivas: la primera, que consistía en denunciar la ineficacia institucional del Estado; y la segunda, en hacer aparecer el tema de la inseguridad pública en los medios de comunicación. Esto último se dio porque la delincuencia,

\footnotetext{
${ }^{17}$ Este consejo estaba integrado por 24 personas: 13 integrantes provenían de diversas instituciones públicas; cuatro de las universidades privadas; uno de la Universidad de Guadalajara; dos de los organismos empresariales; y cuatro de la sociedad civil. Artículo 29 de la Ley de Seguridad Pública del Estado de Jalisco.
} 
Cuadro 5. El proceso de formulación de las políticas de seguridad en Jalisco

\begin{tabular}{l|l|c}
\hline \multicolumn{1}{c|}{ Fase } & \multicolumn{1}{|c|}{ Factores sociopolíticos } & Temporalidad \\
\hline El surgimiento del problema & $\begin{array}{l}\text { El incremento de los índices delictivos y el sentimiento de una creciente inseguridad se apoderó } \\
\text { de la población. }\end{array}$ & $1994-1997$ \\
\hline Discusión política & $\begin{array}{l}\text { Ciertos acontecimientos y condiciones concretas crearon el entorno de discusión entre } \\
\text { empresarios, medios de comunicación y gobierno. }\end{array}$ & $1997-1998$ \\
\hline $\begin{array}{l}\text { Integración y articulación } \\
\text { de demandas }\end{array}$ & $\begin{array}{l}\text { El actor consultado para las distintas modificaciones y para el diseño de las políticas y los } \\
\text { programas del gobierno del estado se concentró básicamente en el sector empresarial. }\end{array}$ & $1995-1999$ \\
\hline Reajustes gubernamentales & $\begin{array}{l}\text { Se implementaron una serie de cambios institucionales y organizativos en la administración } \\
\text { pública. }\end{array}$ & $1998-2000$ \\
\hline $\begin{array}{l}\text { Impactos } \\
\text { Si bien hubo una disminución de los índices delictivos, las políticas de seguridad sólo se } \\
\text { dirigieron a administrar el problema. }\end{array}$ & $2001-2005$ \\
\hline $\begin{array}{l}\text { Reformulación de la agenda } \\
\text { las estrategias }\end{array}$ & $\begin{array}{l}\text { Se mantuvo la percepción de inseguridad y las políticas se dirigieron a lograr la transformación } \\
\text { del Poder Judicial y del sistema de justicia. }\end{array}$ & $2003-2006$ \\
\hline
\end{tabular}

FUENTE: Elaboración propia.

por un lado, aprovechó el anacronismo y la corrupción con que operaban los cuerpos policiacos, así como la dispersión que había en las acciones de las distintas instituciones de seguridad; y porque el empresariado, por el otro, presionaba a las autoridades del estado para que efectuaran una serie de cambios institucionales e implemendelincuencia.

A partir de ese momento, los empresarios se dieron cuenta de su nuevo papel como actores políticos. En la medida en que contaban con la cantidad y calidad de información, la habilidad y el conocimiento para poner recursos y exigir cambios institucionales y organizativos, a diferencia de otros actores (Majone, 2000: 141), trataron de presentarse ante la opinión pública como los actores efectivos en la exigencia de seguridad en la arena política.

El Poder Ejecutivo hizo suyas las demandas de este sector y llevó a cabo las transformaciones burocráticas requeridas. Quedó en evidencia que los empresarios marcaron el ritmo del subsistema de políticas de seguridad y los temas de la agenda institucional. Sin embargo, cuando el gobierno de Jalisco atendió y cumplió cada una de las demandas y vio que no se podía hacer más, las críticas y las exigencias por el entorno de inseguridad se canalizaron hacia el Poder Judicial ${ }^{18}$.

${ }^{18}$ En junio de 1999 diversas cámaras empresariales, organizaciones

\section{Las fases de formulación de las políticas de seguridad pública}

Los argumentos vertidos hasta ahora sostienen la idea de que los empresarios fueron los principales actores en el subsistema de políticas. A partir de su visión del fenómeno se trazaron los alcances técnicos y políticos de la acción gubernamental. En este contexto se constituyó un proceso de formulación de las políticas de seguridad en Jalisco (véase cuadro 5), que constó de las siguientes fases $^{19}$ :

civiles como: México Unido contra la Delincuencia y Mujeres por México entregaron al entonces presidente del Supremo Tribunal de Justicia, Raúl Acosta Cordero, un pliego con las siguientes demandas: 1) que todos los funcionarios del Poder Judicial recuperen su valor ético y moral, su compromiso con la sociedad y la justicia; 2 ) modificación integral a la Ley Orgánica y sus reglamentos para evitar la discrecionalidad al momento de que el magistrado resuelva un asunto; 3) mayor combate a la corrupción e impunidad; 4) depuración de los malos elementos del órgano judicial; 5) capacitación a magistrados, jueces, secretarios y actuarios; 6) que se instaure la carrera judicial; 7) mayor coordinación entre el Poder Judicial y la Procuración del Estado; y 8) mayor coordinación entre el Poder Judicial y el Poder Legislativo. Véase "Demandan sanear al Poder Judicial", Mural, Guadalajara, 22 de junio de 1999, en <www.mural.com>.

19 Para dar orden y claridad al análisis, se diseñó una tipología cronológica de las fases del proceso de actuación pública e intervención del gobierno de Jalisco en el problema de la inseguridad. Los límites temporales de algunas fases no son tajantes, razón por la cual puede haber una imbricación de fases en el proceso de formulación de las políticas de seguridad en Jalisco. El análisis empírico que permitió esta construcción se encuentra en Arellano Ríos, 2004: 106-140. 
El surgimiento del problema. Esta fase se suscita en los años de 1994-1997, después de que las acciones colectivas en torno a la problemática de inseguridad contribuyesen al cambio político, pero sobre todo a que la inseguridad pública emergiera como una preocupación ante el incremento de los índices delictivos. En ella los actores políticos identificaron y constataron que existía un problema que merecía una intervención gubernamental, de tal forma que tuvieron que detallar el significado del problema, precisar su naturaleza e identificar las acciones a implementar (Majone, 2000: 123).

Los índices de robos de vehículos, asaltos bancarios, robos a autotransportes, delitos que conmovieron con mayor fuerza a la clase media, contribuyeron a conformar una base de presión que fue canalizada por los empresarios. Al sentimiento de zozobra por la creciente ola delictiva que se estaba apoderando de la población, se sumó el incremento alarmante del secuestro. Con base en este último, los empresarios señalaron el entorno de indefensión, situación que se reforzó con los plagios de prominentes empresarios; por ejemplo, el ex presidente de la Canaco, René Rivial, y otros empresarios como Antonix Antonio, Federico del Toro, Daniel Castellanos León, Eduardo Valencia Uribe, Emiliano Santana Alencaster y Alfredo Rosales Ruvirosa, entre otros.

La segunda fase del proceso de formulación de las políticas de seguridad en Jalisco, fue la de la discusión politica que se intensificó entre 1997 y 1999. En esta fase el posicionamiento político y mediático del empresariado fue importante. Las críticas y los severos cuestionamientos de las cúpulas empresariales y los medios de comunicación a propósito de la ineficacia de los cuerpos policiacos y de procuración de justicia, así como la ausencia de estrategias en materia de seguridad, constituyeron el punto esencial de la definición del problema y de la actuación gubernamental.

Es en esta parte de la formulación de las políticas de seguridad que el empresariado tuvo los mayores roces públicos con el gobierno del estado; por ejemplo, la declaración conjunta que hizo en la clausura del II Congreso Empresarial de Jalisco en septiembre de 1998. En dicho encuentro, los organismos de la llamada iniciativa privada exigieron la plena vigencia del estado de derecho.
Otro roce fue patente en la rueda de prensa en mayo de 1999, en la que la cúpula empresarial criticó fuertemente al gabinete de seguridad y al Poder Judicial, y exigió la remoción de los titulares de la Secretaría de Seguridad Pública y de la Procuraduría de Justicia ${ }^{20}$. Esta rueda de prensa fue notable y tuvo repercusiones políticas, pues nunca antes se había dado un posicionamiento público de tales características en Jalisco.

La tercera fase fue la de integración y articulación de demandas. De alguna manera siempre estuvo presente durante todo el proceso de formulación de las políticas de seguridad, pero fue en el periodo que va de 1995 a 1999 cuando más se intensificó. En esta fase los empresarios plantearon de manera precisa sus demandas en foros públicos y llevaron a cabo acciones de movilización política con vista a obtener cambios en la acción gubernamental.

Los empresarios, que se habían mostrado alarmados ante el gobierno del estado por el aumento de los índices delictivos y la creciente sensación colectiva de inseguridad, ahora supieron utilizar los mecanismos de presión a su alcance para moldear las políticas públicas. Así, en un primer momento se reestructuró el plan de seguridad pública que hasta entonces había implementado el gobierno de Jalisco. Unos años antes el gobierno estatal había puesto en marcha una nueva legislación y el programa nacional en la materia; sin embargo, estas acciones fueron consideradas como insuficientes ${ }^{21}$. Lo anterior obligó a que en la segunda mitad del sexenio de Alberto Cárdenas los empresarios exigieran al gobierno de Jalis-

\footnotetext{
${ }^{20}$ La rueda de prensa empresarial fue organizada por el CEJ y mostró claramente la intención política de hacer presión para incidir en las decisiones gubernamentales. En esa conferencia de prensa, la cúpula empresarial no sólo denunció sino que exigió "la caída de cabezas" del gabinete de seguridad. Véase "Cambios drásticos en la PGJE, exigen empresarios", Público, 6 de mayo de 1999, en <www.milenio. mx>.

${ }^{21}$ La legislación aludida es la que se refiere a la creación del Sistema de Coordinación de Seguridad Pública en abril de 1994. Este sistema fue duramente criticado por carecer de sustento constitucional, pues en él se integró a las fuerzas militares en funciones de seguridad pública y violaba las disposiciones del artículo $21^{\circ}$ constitucional. Para remediar el vacío jurídico se reformó dicho artículo en el mes de diciembre de 1994. Asimismo, se creó la Ley Reglamentaria de Coordinación del Sistema Nacional de Seguridad Pública en diciembre de 1995. Dicho sistema tuvo como sustento la Ley General que Establece las Bases de Coordinación del Sistema Nacional de Seguridad Pública, véase $<$ www.camaradediputados.mx $>$.
} 
co diseñar un segundo programa: el Programa Jalisco de Seguridad Pública y Procuración de Justicia.

El hecho sociopolítico más importante que se dio en esta fase de formulación de las políticas de seguridad fue la reunión de "alto nivel" entre el gobernador Alberto Cárdenas y la cúpula empresarial22. El gobierno, para dar una respuesta a los empresarios por el delito de los secuestros, creó la Unidad de Atención a Casos Especiales (UACE). Por su parte, el Congreso incrementó las penas para castigar este ilícito ${ }^{23}$. En otra trinchera, los empresarios se confrontaron con organismos civiles y de protección de los derechos humanos. Exigieron espacios en el Consejo General de la Comisión Estatal de Derechos Humanos y denunciaron que la institución "protegía a los delincuentes" y no a las víctimas del delito.

En los años de 1998-2000 se suscitó la cuarta fase del proceso de formulación de las políticas de seguridad pública en Jalisco, que bien podría denominarse como de reajustes gubernamentales. En esta etapa se rediseñaron y modificaron las estrategias gubernamentales de cambio institucional y organizativo. En este orden, el gobierno de

${ }^{22}$ La reunión de "alto nivel" se llevó a cabo a puerta cerrada en Casa Jalisco y duró cuatro horas y media. Se dieron cita los empresarios y el gobernador Alberto Cárdenas, sin la presencia del procurador y del secretario de Seguridad Pública. Al diálogo "cara a cara" con el gobernador asistieron Alejandro Elizondo, presidente de la Canaco; Guillermo Martínez Mora, presidente de Coparmex; Manuel Álvarez Bermejillo, presidente del Consejo de Comercio Exterior de Occidente; José Rubio Torres, presidente del Consejo Agropecuario de Jalisco; Gonzalo Rojas Ramos, presidente del Centro Bancario, y José Luis Macías Romano, coordinador del Consejo de Cámaras Industriales de Jalisco. Véase "Inseguridad, un tema que se discutió tras puertas cerradas", Público, Guadalajara, 18 de mayo de 1999, p. 8.

${ }^{23} \mathrm{El}$ ambiente enrarecido que desataron los secuestros tuvo como consecuencia una inusual protesta de los dirigentes empresariales. Agrupados en el movimiento México Unido contra la Delincuencia y encabezados por el entonces presidente de la Canaco, Xavier Orendáin, se manifestaron en el Congreso de Jalisco con pancartas que decían: "Exigimos leyes más severas contra delincuentes" y pidieron a los diputados que se dictaminara la llamada "Ley Antisecuestros". También acudieron Guillermo Martínez Mora, entonces titular de Coparmex y presidente de México Unido contra la Delincuencia-Jalisco, así como Marisela Moguel, del Círculo de Mujeres por México. Pese a que el acto se desarrolló sin sobresaltos, Xavier Orendáin fue enérgico frente a los diputados. Véase "Empresarios exigen avalar ley antisecuestros", Público, Guadalajara, 4 de diciembre de 1998; y "Aprobaron la ley antiplagios", Público, Guadalajara, 31 de diciembre de 1998, en <www.milenio.com>

de los cuerpos de seguridad y puso en marcha los lineamientos del Programa Jalisco de Seguridad Pública y Procuración de Justicia.

Con base en la idea de modernizar jurídica, administrativa y tecnológicamente a las instituciones de seguridad pública se reformaron los Códigos Penal y de Procedimientos Penales; la Ley Orgánica del Poder Ejecutivo y del Poder Judicial; la Ley Orgánica de la Procuraduría de Justicia; la Ley de Seguridad Pública del Estado y se creó el Consejo Estatal de Seguridad Pública ${ }^{24}$. Al mismo tiempo, se inyectaron cuantiosos recursos presupuestales a las dependencias públicas del sistema judicial. Estas reformas y acciones estatales tuvieron como marco la Ley General que Establece las Bases de Coordinación del Sistema Nacional de Seguridad Pública y la Ley Federal contra la Delincuencia Organizada que impulsó el gobierno de Zedillo en la llamada Cruzada Nacional contra el Crimen y la Delincuencia. También se transformó la Procuraduría General de Justicia. Se creó el Consejo General del Poder Judicial, la Secretaría de Seguridad Pública, Prevención y Readaptación Social, así como el Instituto Jalisciense de Ciencias Forenses.

La quinta fase sería conocida como de impactos. Si bien se presentó una disminución de los índices delictivos entre 2001 y 2005, en los actores y la sociedad en general persistió una sensación de inseguridad e insatisfacción. La aparente eficacia en el combate contra la delincuencia y procuración de justicia del gobierno de Francisco Ramírez, según las cifras oficiales, no fue aparejada con una aprobación satisfactoria de la actuación gubernamental.

Se dio entonces una situación en la cual el gobierno conseguía buenos resultados en su lucha contra la delincuencia pero que la población no percibía. El desencuentro entre la seguridad objetiva y la subjetiva fue más claro cuando Francisco Ramírez presentó su tercer informe de gobierno. En tal escenario, el gobernador, por un lado, reiteró con cifras su percepción técnico-política de que

\footnotetext{
24 Además de este consejo estatal se crearon, en el año 2001, el Consejo Ciudadano de Seguridad Pública del Estado, los Consejos Ciudadanos de Seguridad de Guadalajara y Zapopan, los 13 consejos regionales de seguridad y los 33 comités municipales de seguridad pública. Memorias 1998-2000 del Consejo Ciudadano de Seguridad Pública, Prevención y Readaptación Social, p. 21.
} 
los delitos que preocupaban a los jaliscienses habían disminuido: robo a negocios, a vehículos, a autotransportes, a casa habitación y secuestros; mientras que, por el otro, los demás actores políticos y de la sociedad consideraban que la inseguridad era uno de los reclamos no resueltos ${ }^{25}$.

Descritas las fases anteriores, el proceso de formulación de las políticas de seguridad en Jalisco se dirigió a una sexta fase: la reformulación de la agenda y las estrategias. En ella los actores colocaron ahora como tema central en la agenda de seguridad pública la modernización del sistema de impartición de justicia. El gobierno de Francisco Ramírez se abocó a intentar realizar cambios institucionales con el fin de transformar la Procuraduría de Justicia de Jalisco, instaurar los juicios orales y transparentar el sistema de justicia. Pero al finalizar su sexenio no se concretizó ningún cambio en este sentido.

Recapitulando, aunque los gobiernos panistas pusieron en marcha diversas acciones de cambio y reajuste institucional y organizativo, al final no resolvieron el problema de la inseguridad. La acción gubernamental sólo se limitó a implementar programas de seguridad en un nivel administrativo y técnico. Se conformaron políticas de seguridad pública que descansaban en una serie de intereses, ideas y afinidades ideológicas que criminalizaban la diferencia, la pobreza o la diversidad. En síntesis, eran excluyentes y marginaban.

Algunos empresarios antes críticos, y que denunciaron enérgicamente la actuación del primer gobierno panista y sus fracasos en materia de seguridad pública, ahora formaban parte de él. Pero como tenían puestos públicos y su mirada se centró en hacer carrera política o llenar espacios de organismos autónomos y ciudadanos, ya no continuaron la línea crítica ${ }^{26}$.

${ }^{25}$ Sergio René de Dios, "Dos percepciones de la inseguridad pública", Público, 4 de febrero de 2004, en <www.milenio.com>.

${ }^{26}$ Un primer caso fue el de Guillermo Martínez Mora. Cuando fue presidente de Coparmex era un severo crítico del gobierno de Alberto Cárdenas, pero cuando Francisco Ramírez lo llevó al gabinete estatal su actuación pública cambió radicalmente. Un segundo caso fue el de Alejandro Elizondo quien, como presidente de la Canaco, fue muy activo al exigir al gobierno del Estado Mayor seguridad, pero al igual que en el caso de Martínez Mora, Francisco Ramírez lo promovió ante el Congreso y fue designado presidente del Consejo Electoral de
Los gobiernos, si bien hicieron cambios en sus respectivos espacios institucionales, no lograron resolver el problema de la inseguridad. Se construyó una lógica de reproducción burocrática "perversa", según la cual no se buscó resolver el problema de la inseguridad pública, sino administrarlo. Por lo tanto, el fracaso en materia de seguridad pública en el segundo sexenio panista fue trasladado a otros actores y espacios institucionales como la Federación, el Poder Judicial — ya fuese el local o federal $-\mathrm{u}$ otros estados.

\section{PARA CONCLUIR}

El fenómeno de la inseguridad pública fue un motor inicial del cambio político en Jalisco, que se manifestó en la alternancia, pero una vez lograda ésta, la inseguridad pública pasó a ser sólo un tema de políticas públicas porque el entorno de inseguridad pública — que fue más grave en los gobiernos panistas- dejó de ser un factor de movilización social y política.

En el proceso de formulación de las políticas de seguridad sólo estuvieron —y están presentes- el gobierno de Jalisco y un grupo específico relevante: los empresarios, así como otros actores siempre y cuando respondan a un perfil ideológico común. Esta realidad colocó al fenómeno en la perspectiva de las políticas públicas y la acción gubernamental, y de manera consecuente con ésta, el problema de las políticas de seguridad se conformó más como un problema de legitimidad en el proceso instrumental de la formulación. Los cuestionamientos sobre la legitimidad de las políticas de seguridad fueron más intensos cuando los empresarios luchaban por ser incluidos.

Así, el gobierno puso atención a sus demandas y rediseñó a partir de su visión un complejo organizativo e institucional. De esta forma, el empresariado fue el interlocutor efectivo en la medida en que marcó la agenda del cambio institucional en materia de seguridad pública y de los espacios en los cuales se debió actuar. Ello implicó

Jalisco. La integración de empresarios antes críticos tuvo como estrategia quitar el "ruido" mediático y allegarse del apoyo de la cúpula empresarial. 
avances en un principio, pero posteriormente estancó las políticas de seguridad.

Consecuentemente, las políticas de seguridad pública no fueron claras ni constantes en su cometido, pues la inseguridad pública siguió siendo un problema que no resolvían los gobiernos de cualquier filiación partidista. Se llegó así al escenario en el que las políticas que aspiraron a ser públicas se convirtieron en políticas gubernamentales o, en el peor de los casos, a ser sólo programas burocráticos. No fueron políticas públicas, por un lado, porque carecieron del elemento de la participación ciudadana (elemento clave para ser consideradas como tales).Y por el otro, porque tendieron más a la lógica burocrática, con la consecuencia de que la acción gubernamental se ocupara sólo de administrar el problema y reproducir interacciones burocráticas sin sentido.

\section{Bibliografía}

Acosta Silva, Adrián, 2004, "Poder político, alternancia y desempeño institucional. La educación superior en Jalisco, 1995-2001”, Estudios Sociológicos, vol. XXII, núm. 64, enero-abril, El Colegio de México, México, pp. 54-55.

Alba, Carlos y Dirk Kruijt, 1988, Los empresarios y la industria de Jalisco, El Colegio de Jalisco, Zapopan.

—_ y Fernando González, 1989, "Dirigentes económicos y poderes regionales en Jalisco”, en Edmundo Jacobo, Matilde Luna y Ricardo Tirado (coords.), Empresarios de México, Universidad de Guadalajara, Guadalajara, pp. 381-394.

Alonso, Jorge, 1995, El cambio en Jalisco. Las elecciones en 1994 y 1995, Universidad de Guadalajara y Centro de Investigaciones, Estudios Superiores en Antropología Social, México, Guadalajara.

Aguilar Villanueva, Luis F., 1996, "Estudio introductorio", en Problemas públicos y agenda de gobierno, Miguel Ángel Porrúa, México, pp. 15-72.

Arellano Ríos, Alberto, 2004, Empresarios e inseguridad pública en Jalisco: la formulación de las políticas de seguridad, tesis de maestría, Instituto Tecnológico de Estudios Superiores de Occidente, Tlaquepaque.

Arraigada, Irma y Lorena Godoy, 1999, Seguridad ciudadana y violencia en América Latina, diagnóstico y políticas en los años noventa, núm. 32, agosto, CEPAL, Santiago de Chile.
Buvinic et al., 1999, "Violence in Latin America and the Caribbean: A Framework for Action", informe técnico, Banco Interamericano de Desarrollo, Washington.

Cohen, Jean L. y Andrew Arato, 2000, Sociedad civil y teoría política, Fondo de Cultura Económica, México.

Frühling, Hugo, 2002, "Policía y sociedad. Tres experiencias sudamericanas", Renglones, núm. 51, mayo-agosto, Instituto Tecnológico de Estudios Superiores de Occidente, Tlaquepaque, pp. 23-35.

Gómez López, Alicia, 1997, Crisis y transición en Jalisco, Universidad de Guadalajara, Guadalajara.

Hernández Águila, Elena de la Paz, 2002, "Hacia una nueva cultura empresarial”, en Jorge Regalado Santillán y Juan Manuel Ramírez Sáiz (coords.), Jalisco antes y después de 1995, Universidad de Guadalajara, Guadalajara, pp. 129-147.

Majone, Giandomenico, 2000, Evidencia, argumentación y persuasión en la formulación de políticas, Fondo de Cultura Económica, México.

Moloeznik, Marcos Pablo, 2002, "Seguridad humana. Un nuevo enfoque impulsado por la ONU”, Renglones, núm. 51, mayo-agosto, Instituto Tecnológico de Estudios Superiores de Occidente, Tlaquepaque, pp. 45-96.

Morlino, Leonardo, 1995, ¿Cómo cambian los regímenes políticos?, Centro de Estudios Constitucionales, Madrid.

Olveda, Jaime, 1991, La oligarquía de Guadalajara, Consejo Nacional para la Cultura y las Artes, México.

Pasquino, Gianfranco et al., 1986, "Participación política, grupos y movimientos", en Manual de ciencia politica, Alianza Universidad, Madrid, pp. 179-215.

Preston, Julia y Samuel Dillon, 2004, "El general y el señor de los cielos", en El despertar de México. Episodios de una búsqueda de la democracia, Océano, México, pp. 207-228.

Regalado Santillán, Jorge, 2001, Sociedad civil y gobierno. La seguridad pública en Jalisco, tesis de doctorado, Centro de Investigaciones y Estudios Superiores en Antropología Social, Universidad de Guadalajara, Guadalajara.

— 2005, "Contradicciones y paradojas de la alternancia política en Jalisco", conferencia en el "Seminario-taller de investigación”, 27 de mayo, El Colegio de Jalisco, Zapopan.

__ y Marcos Pablo Moloeznik, 1998, "Privatización de la seguridad pública. Una aproximación a partir de estudios de caso", en Rigoberto Gallardo y Joaquín Osorio (coords.), México y Jalisco en la coyuntura. Primer semestre de 1998, Instituto Tecnológico de Estudios Superiores de Occidente, Guadalajara, pp. 111-132.

Romero, Laura Patricia, 2001, Jalisco: hacia una democracia gobernable, Universidad de Guadalajara, Guadalajara. 